\title{
Comunicação \\ Armazenamento de sementes de cerejas-do-mato (Eugenia involucrata) DC. submetidas ao recobrimento com biofilmes e embalagem a vácuo
}

\author{
Alexandre Luis Alegretti ${ }^{1}$, Américo Wagner Júnior ${ }^{* 2}$, Aldair Bortolini', Cristiano Hossel', \\ Juliano Zanela, Idemir Citadin ${ }^{2}$
}

http://dx.doi.org/10.1590/0034-737X201562010016

\begin{abstract}
RESUMO
Fruteiras como Eugenia involucrata ainda continuam inexploradas, necessitando-se de informações técnicas que incentivem o agricultor a utilizá-las. O método de propagação por sementes é o normalmente adotado; porém, elas devem ser imediatamente semeadas, pois corre-se o risco de perda de sua viabilidade com o armazenamento. $\mathrm{O}$ objetivo deste trabalho foi avaliar o hidrocondicionamento e as técnicas de conservação (vácuo e biofilme), durante armazenamento, aos cinco e aos 30 dias, de sementes de cerejeira-do-mato. O trabalho foi realizado na Universidade Tecnológica Federal do Paraná - Câmpus Dois Vizinhos. O delineamento experimental foi em blocos ao acaso, em fatorial $2 \times 4 \times 2$ (pré-hidrocondicionamento $\mathrm{x}$ técnica de armazenamento $\mathrm{x}$ tempo de armazenamento), com quatro repetições, de 50 sementes por unidade experimental. As sementes extraídas foram separadas em dois lotes, sendo um submetido ao pré-hidrocondicionamento, em água destilada, durante 24 horas, e, outro, não. Sementes hidrocondicionadas, ou não, foram submetidas a quatro técnicas de armazenamento, sendo, estas, a embalagem plástica a vácuo, o revestimento com biofilme de fécula de mandioca $(3 \% \mathrm{~m} / \mathrm{v})$, a embalagem plástica a vácuo + biofilme de fécula de mandioca e sem tratamento (controle). Posteriormente, as sementes foram mantidas em câmara fria, em temperatura de $5^{\circ} \mathrm{C}$ e UR $85 \%$, durante cinco e 30 dias. Aos 110 dias após a semeadura, avaliou-se a percentagem de germinação, o índice de velocidade de emergência e a massa da matéria seca total das plântulas. Para o armazenamento das sementes de cerejeira-do-mato, devem-se utilizar técnicas a vácuo, isoladamente, ou com revestimento de biofilme.
\end{abstract}

Palavras-chave: conservação, Eugenia sp., Myrtaceae.

\section{ABSTRACT}

\section{Biofilms and vacuum package in the storage of Eugenia involucrata DC. seeds}

Fruit trees such as Eugenia involucrata are still unexploited, therefore technical information that encourages farmers to use them is needed. The seed propagation method is usualy used since there is the risk of losing its viability with storage. The objective of this work was to evaluate the hydropriming and storage techniques (vacuum and biofilm), during storage at five and 30 days of E. involucrata seeds. The study was carried out at Universidade Tecnológica Federal do Paraná - Dois Vizinhos campus, Paraná State, Brazil. The experimental design was a randomized block, in the $2 \times 4 \times 2$ factorial design (hydropriming x storage technique x storage time), with 4 replications of 50 seeds per plot. The extracted seeds were separated into two groups: one was submitted to prehydropriming in distilled water for 24 hours and the other not. Hydroprimed or not-hydroprimed seeds were submitted to four storage techniques: vacuum plastic packaging, cassava starch biofilm seed coated (3\% w/v), vacuum plastic

\footnotetext{
Submetido em 04/10/2012 e aprovado em 17/09/2014.

${ }^{1}$ Universidade Tecnológica Federal do Paraná, Dois Vizinhos, Paraná, Brasil. alexandreluisalegretti@ yahoo.com.br

Universidade Tecnológica Federal do Paraná, Dois Vizinhos, Paraná, Brasil. americowagner@utfpr.edu.br; idemir@utfpr.edu.br (Bolsista de Produtividade CNPq).

${ }^{3}$ Universidade Tecnológica Federal do Paraná, Dois Vizinhos, Paraná, Brasil. aldair.b1@hotmail.com

${ }^{4}$ Universidade Tecnológica Federal do Paraná, Dois Vizinhos, Paraná, Brasil. julianoz@utfpr.edu.br

Autor para correspondência: americowagner@utfpr.edu.br
} 
packaging + cassava starch biofilm and no treatment (control). Afterwards, the seeds were kept in a cold chamber at $5^{\circ} \mathrm{C}$ and $85 \%$ relative humidity for five and 30 days. One hundred and ten days after sowing, the germination percentage, emergence speed index and total dry matter mass of the plantlets were evaluated. For storage of E. involucrata seeds, the vacuum technique with or without biofilm coating should be used.

Key words: Eugenia sp., Myrtaceae, storage.

\section{INTRODUÇÃO}

O Brasil destaca- se por ser um dos principais centros de diversidade genética de fruteiras nativas. Contudo, algumas espécies estão desaparecendo da natureza, antes mesmo que se tenha conhecimento básico de sua biologia e de seu potencial (Laundry \& Kawasaki, 1997).

O potencial das fruteiras da família Myrtaceae são inúmeros, uma vez que podem ser utilizadas em programas de recuperação de áreas degradadas e de preservação permanente, por terem frutos amplamente consumidos pela avifauna, o que auxilia na dispersão das sementes (Lorenzi, 2002), além de apresentarem propriedades funcionais, contribuindo para a saúde do homem (Rocha et al., 2011).

Dentre todos os gêneros de espécies frutíferas dessa família, apenas cinco (Eugenia, Acca, Myrthacea, Plinia e Psidium), atualmente, têm importância econômica (Danner et al., 2010), uma vez que abrangem espécies que produzem frutos com características sensoriais aceitáveis. Porém, apesar desta potencialidade, fruteiras como a cerejeira-do-mato (Eugenia involucrata DC. - Myrtaceae) ainda continuam praticamente inexploradas, pela falta de informações técnicas que incentivem o agricultor a utilizá-las em sua propriedade. Neste sentido, tornam-se necessários estudos que contribuam para sua manutenção e perpetuação, principalmente aqueles ligados a sua propagação.

A cerejeira-do-mato é propagada principalmente por via seminífera, podendo também utilizar os métodos assexuados, como estaquia e enxertia. Entretanto, estes métodos, quando testados, não apresentaram resultados satisfatórios de enraizamento e pegamento, respectivamente (Pirola, 2013).

Todavia, apesar de muito usadas, as sementes apresentam limitações ao uso, já que, nesta espécie, mostram baixo poder germinativo quando armazenadas por períodos superiores a 30 dias (Lorenzi, 2002), pelo fato de serem classificadas como recalcitrantes.

Esse baixo poder germinativo das sementes recalcitrantes, quando armazenadas, deve-se a fatores ligados aos danos causados ao embrião durante o processo de extração e dessecação, pelas variações de temperatura e, também, como necessitam ser armazenadas com alto teor de umidade, pela facilidade de contaminação microbiana e a própria germinação durante o período de armazenamento (Chin \& Roberts, 1980).

Assim, técnicas devem ser testadas, visando a prolongar a viabilidade dessas sementes quando armazenadas. Dentre estas, têm-se o hidrocondicionamento, que permite hidratar as sementes, preparando seu metabolismo para o processo de germinação, sem que ocorra a emissão da raiz primária (Giurizatto et al., 2008), tornando-o útil para sua conservação.

Aliadas a esta técnica, podem-se utilizar embalagens plásticas com vácuo, ou, o revestimento das sementes com biofilmes, já que ambas as técnicas também podem contribuir para aumentar a viabilidade das sementes, uma vez que reduzem sua atividade metabólica pela limitação das trocas gasosas com o meio externo, impossibilitando sua germinação e ao mesmo tempo mantendo-as conservadas.

O objetivo deste trabalho foi avaliar o efeito do hidrocondicionamento e de técnicas de conservação (vácuo e biofilme) no armazenamento de sementes de cerejeira-domato.

\section{MATERIAL E MÉTODOS}

O trabalho foi realizado em dois ambientes, sendo parte no Laboratório de Fisiologia Vegetal e, outra, na Unidade de Ensino e Pesquisa Viveiro de Produção de Mudas, da Universidade Tecnológica Federal do Paraná - Campus Dois Vizinhos (PR).

$\mathrm{O}$ delineamento experimental utilizado foi em blo$\cos$ ao acaso, em esquema fatorial $2 \times 4 \times 2$ (préhidrocondicionamento $\mathrm{x}$ técnica de armazenamento $\mathrm{x}$ tempo de armazenamento), com quatro repetições de 50 sementes por unidade experimental.

As sementes foram extraídas de frutos maduros de cerejeira-do-mato. Em seguida, elas foram separadas em dois lotes, sendo o primeiro submetido ao processo de préhidrocondicionamento em água destilada, em temperatura ambiente, com embebição durante 24 horas e o segundo não foi hidrocondicionado em água destilada. Em seguida, sementes hidrocondicionadas, ou não, foram submetidas a quatro técnicas de armazenamento, sendo estas embalagem plástica a vácuo, revestimento com biofilme de 
fécula de mandioca $(3 \% \mathrm{~m} / \mathrm{v})$, embalagem plástica a vácuo + revestimento com biofilme de fécula de mandioca e sem revestimento e uso de embalagem plástica.

Posteriormente, as sementes foram mantidas em câmara fria, em temperatura de $5{ }^{\circ} \mathrm{C}$ e umidade relativa (UR) $80 \%$, durante cinco e 30 dias. Após cada período, as sementes foram semeadas em canteiros, utilizandose areia como substrato. As irrigações foram feitas em dois turnos de 30 minutos cada (início da manhã e final da tarde), sempre verificando-se previamente a umidade do substrato. Aos 110 dias após a semeadura, avaliaramse a percentagem de emergência, o índice de velocidade de emergência (IVE) e a massa da matéria seca total das plantas. Os dados foram submetidos à análise de variância e comparação de médias pelo teste de Tukey $(\mathrm{p}<0,05)$. Os dados da percentagem de emergência foram transformados previamente em arco seno $\sqrt{x / 100}$, uma vez que o teste de normalidade de Lilliefors demonstrou sua necessidade. As análises estatísticas foram realizadas no software SANEST ${ }^{\circledR}$ (Zonta \& Machado, 1984).

\section{RESULTADO E DISCUSSÃO}

Não houve interação significativa entre os fatores pré-hidrocondicionamento $\mathrm{x}$ técnica de armazenamento $\mathrm{x}$ tempo de armazenamento, bem como, pré-hidrocondicionamento $\mathrm{x}$ técnica de armazenamento, préhidrocondicionamento $\mathrm{x}$ tempo de armazenamento para emergência, IVE e massa da matéria seca das plantas. Porém, a interação entre armazenamento $\mathrm{x}$ tempo de armazenamento mostrou-se estatisticamente significativa (Tabela 1), bem como, para o fator tempo de armazenamento, nas três variáveis analisadas (Tabela 2).

De acordo com a Tabela 1, observou-se que as sementes sem revestimento e embalagem a vácuo (controle) e, com uso de biofilme de fécula de mandioca, proporcionaram plântulas com maior massa de matéria seca das plantas, quando armazenadas durante cinco dias, se comparadas com aquelas armazenadas por 30 dias. Por outro lado, quando se utilizou a técnica de embalagem plástica a vácuo, com e sem biofilme de fécula de mandioca, verificaram-se médias de massa de matéria seca de plantas iguais, estatisticamente, para as sementes mantidas durante cinco e 30 dias de armazenamento.

O fato de as plântulas do tratamento controle terem sido superiores para massa de matéria seca, aos cinco dias, em comparação com aquelas dos 30 dias, pode ser em decorrência do maior consumo das reservas das sementes armazenadas por 30 dias, pois, para manteremse viáveis por maior período, é necessário o fornecimento das reservas contidas em seu interior, reduzindose o vigor, a posteriori, das plântulas. O mesmo deve ter ocorrido para superioridade das sementes revestidas com biofilme aos cinco dias.
Analisando-se as variáveis dentro do fator técnica de armazenamento (Tabela 2), observaram-se as maiores médias de emergência com embalagem a vácuo, tendo ou não o revestimento das sementes com biofilme de fécula de mandioca, sendo esses valores superiores a $82 \%$. O mesmo resultado de superioridade destes tratamentos também foi observado para o IVE, demonstrando-se efeito benéfico do uso de vácuo para conservação das sementes de cerejeira-do-mato.

O mesmo efeito benéfico do uso de embalagem a vácuo foi demonstrado por Danner et al. (2011) e Hossel et al. (2013), com jabuticabeira, outra Myrtaceae recalcitrante. Neste sentido, verifica-se efeito positivo do uso da embalagem a vácuo para conservação das sementes, supondo-se que o uso dessa técnica não permite as trocas gasosas, diminuindo-se a atividade metabólica das sementes, o que acarreta, consequentemente, a preservação de maior quantidade de reservas, possibilitando a permanência do vigor das plântulas, posteriormente à sua germinação.

Já o fator pré-hidrocondicionamento das sementes não afetou significativamente as variáveis emergência, IVE e massa da matéria seca das plântulas, mostrando não ser necessário seu uso para o armazenamento de sementes de

Tabela 1. Massa de matéria seca (g) de plântulas de cerejeirado-mato provenientes de sementes armazenadas por 5 e 30 dias a $5{ }^{\circ} \mathrm{Ce} 80 \%$ de UR, submetidas a diferentes tipos de recobrimento

\begin{tabular}{lcc}
\hline \multirow{2}{*}{ Técnica } & \multicolumn{2}{c}{ Tempo (Dias) } \\
\cline { 2 - 3 } & $\mathbf{5}$ dias & $\mathbf{3 0}$ dias \\
\hline Controle & $2,79 \mathrm{a} \mathrm{A*}$ & $1,34 \mathrm{~b} \mathrm{BC}$ \\
Biofilme de fécula & $2,66 \mathrm{a} \mathrm{A}$ & $0,76 \mathrm{~b} \mathrm{C}$ \\
de mandioca & $2,66 \mathrm{a} \mathrm{A}$ & $2,73 \mathrm{a} \mathrm{AB}$ \\
Vácuo & $4,01 \mathrm{a} \mathrm{A}$ & $2,81 \mathrm{a} \mathrm{A}$ \\
Biofilme de fécula & & \\
de mandioca + Vácuo & & \\
\hline
\end{tabular}

CV (\%) 50,87

*Letras minúsculas na mesma linha e maiúsculas na coluna diferem entre si estatisticamente pelo teste Tukey $(p<0,05)$.

Tabela 2. Germinação e IVE de sementes de cerejeira-do-mato armazenadas por 5 e 30 dias a $5^{\circ} \mathrm{C}$ e $80 \%$ de UR, submetidas a diferentes tipos de recobrimento

\begin{tabular}{lcc}
\hline Técnica & Germinação (\%) & IVE \\
\hline Controle & $75,61 \mathrm{~b}^{*}$ & $2,06 \mathrm{bc}$ \\
Biofilme de fécula & $73,39 \mathrm{~b}$ & $1,71 \mathrm{c}$ \\
de mandioca & $82,67 \mathrm{ab}$ & $3,19 \mathrm{ab}$ \\
Vácuo & $89,29 \mathrm{a}$ & $3,41 \mathrm{a}$ \\
$\begin{array}{l}\text { Biofilme de fécula } \\
\text { de mandioca + Vácuo }\end{array}$ & 19,75 & 19,51 \\
\hline CV $(\%)$ &
\end{tabular}

*Médias seguidas por letras minúsculas distintas na coluna diferem entre si pelo Teste de Tukey $(p<0,05)$. 
cerejeira-do-mato. Supõe-se que, pelo fato de que sementes, ao serem extraídas, foram em seguida submetidas aos tratamentos de hidrocondicionamento, elas já continham teor de umidade adequada em seu interior, não acarretando assim a significância estatística que estimulasse maior atividade metabólica de suas sementes.

Delgado (2006) descreveu que normalmente o teor de umidade das sementes de cerejeira-do-mato, no momento de sua extração, é de $58 \%$, valor que já permite semeá-la, obtendo-se satisfatória percentagem de emergência.

Quanto ao tempo de armazenamento das sementes, observou-se que todas as variáveis analisadas apresentaram maiores médias quando mantidas até cinco dias, em comparação com as mantidas até 30 dias (Tabela 3), verificando-se que a cerejeira-do-mato reduz seu poder germinativo e seu vigor com o armazenamento, conforme já ressaltado por Maluf et al. (2003) e Pirola (2013). Todavia, ressaltam-se avanços, neste estudo, uma vez que, aos 30 dias, foi possível manter emergência acima de $65 \%$, superior à média obtida por Barbedo et al. (1998), para igual período.

Tabela 3. Germinação de sementes, IVE e massa de matéria seca das plântulas de cerejeira-do-mato armazenadas por 5 e 30 dias a $5^{\circ} \mathrm{C}$ e $80 \%$ de UR, submetidas a diferentes tipos de recobrimento

\begin{tabular}{lccc}
\hline Tempo & Germinação $(\%)$ & IVE & MMS \\
\hline 5 dias & $91,24 \mathrm{a}^{*}$ & $2,98 \mathrm{a}$ & $0,47 \mathrm{a}$ \\
30 dias & $67,16 \mathrm{~b}$ & $2,21 \mathrm{~b}$ & $0,33 \mathrm{~b}$ \\
\hline CV $(\%)$ & 19,75 & 19,51 & 50,87 \\
\hline
\end{tabular}

*Médias seguidas por letras minúsculas distintas na coluna diferem entre si pelo Teste de Tukey $(p<0,05)$.

\section{CONCLUSÃO}

Para conservação das sementes de cerejeira-do-mato devem-se utilizar técnicas a vácuo, isoladamente ou com revestimento de biofilme.

\section{AGRADECIMENTOS}

Os autores agradecem ao Projeto de Extensão da Universidade sem Fronteira, Apoio à Agroecologia, no Sudoeste do Paraná, Plano Trienal 2008/2010, da Assesoar, pela concessão da bolsa e pelo auxílio aos demais autores durante a pesquisa.

\section{REFERÊNCIAS}

Barbedo CJ, Kohama S, Maluf AM \& Bilia DAC (1998) Germinação e armazenamento de diásporos de cerejeira (Eugenia involucrata D.C. - Myrtaceae) em função do teor de água. Revista Brasileira de Sementes, 20:184-188.

Chin HF \& Roberts EH (1980) Recalcitrant crop seed. Malaysia, Tropical Press SDN. BHD. 152p.
Danner MA, Citadin I, Sasso SAZ, Sachet MR \& Ambrosio R (2010) Fenologia da floração e frutificação de Mirtáceas nativas da Floresta com Araucária. Revista Brasileira de Fruticultura, 32:291-295

Danner MA, Citadin I, Sasso SAZ, Ambrosio R \& Wagner Júnior A (2011) Armazenamento a vácuo prolonga a viabilidade de sementes de jabuticabeira. Revista Brasileira de Fruticultura, 33:246-252.

Delgado LF (2006) Tolerância à dessecação em sementes de espécies brasileiras de Eugenia. Dissertação de Mestrado. Instituto de Botânica da Secretaria do Meio Ambiente, São Paulo. 106p.

Giurizatto MIK, Robaina AD, Gonçalves MC \& Marchetti ME (2008) Qualidade fisiológica de sementes de soja submetidas ao hidrocondicionamento. Acta Scientiarum Agronomy, 30:711717

Hossel C, Oliveira JSMA, Fabiane KC, Wagner Júnior A \& Citadin I (2013) Conservação e teste de tetrazólio em sementes de jabuticabeira. Revista Brasileira de Fruticultura, 35:255-261.

Laundry LR \& Kawasaki ML (1997) The genera of Myrtaceae in Brazil: an illustrated synoptic treatment and identification keys. Brittonia, 49:508-536.

Lorenzi H (2002) Árvores Brasileiras: manual de identificação e cultivo de plantas arbóreas nativas do Brasil. $4^{\mathrm{a}} \mathrm{ed}$. Nova Odessa, Instituto Plantarum. 368p.

Maluf AM, Bilia DAC \& Barbedo CJ (2003) Drying and storage of Eugenia involucrata D.C. Seeds. Scientia Agricola, 60:471-475.

Pirola K (2013) Caracterização fisiológica e conservação de sementes de oito fruteiras nativas do Bioma Floresta com Araucária. Dissertação de Mestrado. Universidade Tecnológica Federal do Paraná, Pato Branco. 129p.

Rocha WS, Lopes RM, Silva DB, Vieira RF, Silva JP \& Agostini-Costa TS (2011) Compostos fenólicos totais e taninos condensados em frutas nativas do cerrado. Revista Brasileira de Fruticultura, 33:12151221.

Zonta EP \& Machado AA (1984) Sistema de Análise Estatística (SANEST). Pelotas, UFPEL. 399p. 